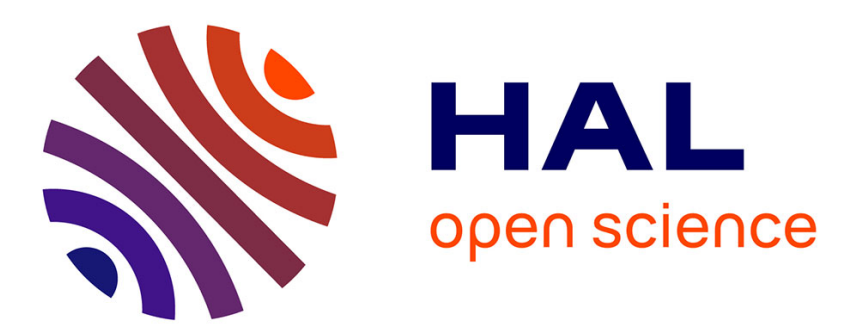

\title{
Indirect adaptive fuzzy control scheme based on observer for nonlinear systems: A novel SPR-filter approach
}

\author{
Abdesselem Boulkroune, N. Bounar, Mohammed M'Saad, Mondher Farza
}

\section{To cite this version:}

Abdesselem Boulkroune, N. Bounar, Mohammed M'Saad, Mondher Farza. Indirect adaptive fuzzy control scheme based on observer for nonlinear systems: A novel SPR-filter approach. Neurocomputing, 2014, 135, pp.Pages 378-387. 10.1016/j.neucom.2013.12.011 . hal-01062734

\section{HAL Id: hal-01062734 \\ https://hal.science/hal-01062734}

Submitted on 15 Sep 2014

HAL is a multi-disciplinary open access archive for the deposit and dissemination of scientific research documents, whether they are published or not. The documents may come from teaching and research institutions in France or abroad, or from public or private research centers.
L'archive ouverte pluridisciplinaire HAL, est destinée au dépôt et à la diffusion de documents scientifiques de niveau recherche, publiés ou non, émanant des établissements d'enseignement et de recherche français ou étrangers, des laboratoires publics ou privés. 


\title{
Indirect adaptive fuzzy control scheme based on observer for nonlinear systems: A novel SPR-filter approach
}

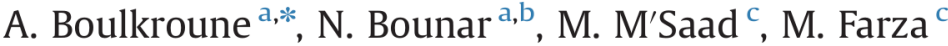 \\ ${ }^{a}$ LAJ, Department of Automatic Control, University of Jijel, BP. 98, Ouled-Aissa, 18000 Jijel, Algeria \\ ${ }^{\mathrm{b}}$ LCP, Department of Automatic Control, Ecole Nationale Polytechnique (ENP), 10, Av. Hassen Badi, BP182, Algiers, Algeria \\ ' GREYC, UMR 6072 CNRS, Université de Caen, ENSICAEN, 6 Bd Maréchal Juin, 14050 Caen Cedex, France
}

Keywords:

Fuzzy system

Indirect adaptive control

Observer

SPR condition

Nonlinear system

\begin{abstract}
A B S T R A C T
In this paper, a novel fuzzy indirect adaptive controller based on observer for uncertain nonlinear perturbed systems is proposed. A tracking-error observer is introduced to resolve the problem of the unavailability of state variables. Adaptive fuzzy systems are employed to approximate the unknown smooth nonlinear functions. The control system is augmented by a low-pass filter designed to meet a SPR condition of a transfer function of the observation error dynamics. The SPR condition is used in the Lyapunov stability analysis to construct the adaptation laws using only available measurements (i.e. the output observation error and the output tracking error). The main contributions of this paper lie in the following: (a) The SPR-filter approach used here avoids the filtering of the fuzzy basis functions. (b) Unlike in the previous works, the stability analysis is rigorously proven by using a SPR-based Lypunov approach. Finally, numerical simulation results are presented to verify the feasibility and effectiveness of the proposed controller.
\end{abstract}

\section{Introduction}

Fuzzy systems (FS) as well as neural networks (NN) have been successfully applied to many control problems because they do not need an accurate mathematical model of the system under control. It is also known that these intelligent systems (i.e. FS and NN) can approximate uniformly any nonlinear continuous function over a compact set [1-3]. Recently, much attention has been focused on adaptive neural/fuzzy control of nonlinear dynamical systems [4-17]. With suitable coordinate transformation, a class of nonlinear dynamic systems can be transformed into the so-called normal form [4-7], or into the cascade triangular forms, which are classified as strict-feedback and pure-feedback forms [8-17]. For the first class [4-7], fuzzy or neural adaptive controls were developed in which uncertainties are well dealt with. The stability of the closed-loop system has been only investigated using a standard Lyapunov approach. For the second class [8-17], fuzzy or neural adaptive backstepping controls were developed in which both uncertainties and non-matching conditions are well dealt with. The stability analysis of these control schemes has been investigated using the backstepping concept and Lyapunov approach.

\footnotetext{
* Corresponding author.

E-mail addresses: boulkroune2002@yahoo.fr (A. Boulkroune), bounar18@yahoo.fr (N. Bounar), msaad@greyc.ensicaen.fr (M. M'Saad), mfarza@greyc.ensicaen.fr (M. Farza).
}

This last class of the nonlinear systems is beyond the scope of this paper. We are interested here to the nonlinear systems having the normal form or which can be transformed into this form. Although the adaptive neural/fuzzy control systems designed in the literature [4-7] for this class of systems are simple and can give satisfactory results, they have certain limitations and shortcomings, namely: (1) the state vector of these systems is assumed to be available for measurement. But this measurement requirement is more an exception than a rule in the engineering practice. That is why observer-based controllers (or output-feedback controllers) are most used in practice. (2) These control systems can only be applied to the nonlinear systems which can satisfy a harsh requirement named matching condition, i.e. the nonlinearities only appear in the same equation as the control in the state space representation.

Based on state or tracking-error observer, direct and indirect adaptive fuzzy control schemes have been developed in [18-24]. These schemes require strictly positive real (SPR) condition on the observation error dynamics (i.e. the estimation error dynamics) so that one can use Meyer-Kalman-Yakubovich (MKY) lemma in the stability analysis. The original observation error dynamics, which are not SPR in general, are augmented by a low-pass filter designed to satisfy the SPR condition of a transfer function associated with the Lyapunov stability analysis. However, according to [25], these schemes result in the filtering of the fuzzy basis function (FBF) which is not generally suitable. Moreover, as stated in [26], these observer-based fuzzy (direct or indirect) adaptive 
controllers have not been derived rigorously in mathematics. In fact, some comments on these control schemes have been made in [26].

In this paper, a novel fuzzy indirect adaptive controller is investigated for a class of uncertain perturbed monovariable nonlinear systems. The main difficulties are how to deal with unknown nonlinear functions, to appropriately design an observer-based indirect adaptive control using the SPR condition and to compensate for the fuzzy approximation errors which depend on the control input. In this paper, these difficulties can be respectively solved by using the fuzzy systems for the function approximation, by introducing an auxiliary error and by designing a new robust dynamic compensator. The main contributions of this paper lie in the following:

- A novel fuzzy indirect adaptive output-feedback control scheme based on SPR condition for nonlinear systems is proposed.

- Unlike in [18-24], there is no filtering of the FBF vectors in this proposed adaptive control scheme.

- By using the SPR condition and Lyapunov theory, the stability of the closed-loop system is rigorously proven. Recall that all previous observer-based fuzzy indirect and direct adaptive control schemes [18-24] have not been derived rigorously in mathematics, as stated in [26].

\section{Problem formulation, preliminaries and fuzzy systems}

\subsection{Problem formulation and preliminaries} form:

Consider the $n$th order nonlinear dynamical system of the

$x^{(n)}=f\left(x, \dot{x}, \ldots, x^{(n-1)}\right)+g\left(x, \dot{x}, \ldots, x^{(n-1)}\right) u+d(t), y=x$

Or equivalently of the form

$\dot{x}=A x+B[f(x)+g(x) u+d(t)], y=C^{T} x$

with

$A=\left[\begin{array}{ccccc}0 & 1 & 0 & \cdots & 0 \\ 0 & 0 & 1 & \cdots & 0 \\ \vdots & \vdots & \vdots & \ddots & \vdots \\ 0 & 0 & 0 & \cdots & 1 \\ 0 & 0 & 0 & \cdots & 0\end{array}\right], B=\left[\begin{array}{c}0 \\ 0 \\ \vdots \\ 0 \\ 1\end{array}\right], C=\left[\begin{array}{c}1 \\ 0 \\ \vdots \\ 0 \\ 0\end{array}\right]$,

where $u \in R$ is the control input, $x=\left[x_{1}, x_{2}, \ldots, x_{n}\right]^{T}=$ $\left[x, \dot{x}, \ldots, x^{(n-1)}\right]^{T} \in R^{n}$ is the vector of unmeasured states and $y \in R$ is the measured output. $f(x)$ and $g(x)$ are unknown smooth functions, $d(t)$ is the external disturbance. Note that the pair $(A, B)$ is controllable and the pair $\left(C^{T}, A\right)$ is observable.

Design objective: Determine an output-feedback Determine an output-feedback control law $u$ to steer the system output $y$ closes to a reference signal $y_{r}$, while ensuring that all involved signals in the closed-loop system remain uniformly ultimately bounded (UUB).

To facilitate control system design, the following usual assumptions are presented and will be used in the subsequent developments.

Assumption 1. There exists an unknown positive constant $d^{*}$ such that $|d(t)| \leq d^{*}$.

Assumption 2. The reference signals $y_{r}, \dot{y}_{r}, \ldots, y_{r}^{(n-1)}$, and $y_{r}^{(n)}$ are assumed to be continuous and bounded.
Assumption 3. There exists an unknown positive constant $g_{0}$ such that: $0<g_{0} \leq|g(x)|[26,27]$.

The following remarks allow to motivate the above assumptions with respect to the considered design framework:

Remark 1. Assumption 1 is usually required in the system theory, e.g. see $[18,22,25,26]$. Assumption 2 is a standard assumption in the adaptive control literature. The latter is the first to be made in an adaptive control scheme and can be given explicitly or implicitly.

Remark 2. Many practical systems can be expressed or transformed in the form (1) such as inverted pendulum system $[3,26]$, Duffing oscillator [3,26], Chua's chaotic circuit [28], massspringer-damper system [29], induction servo-motor system [30], gyro system [31], Genesio-Tesi chaotic system [32], singlelink robot [33], atomic force microscope [34], autocatalysed chemical reaction [35] and many others. Assumption 3 is not restrictive as it is satisfied by all these practical systems. It guarantees the controllability of the system (1).

Let us define the reference signal vector $\underline{y}_{r}$ and the tracking error vector as follows:

$\underline{y}_{r}=\left[y_{r}, \dot{y}_{r}, \ldots, y_{r}^{(n-1)}\right]^{T}, \quad \underline{e}=\underline{y}_{r}-\underline{x}=\left[e_{1}, e_{2}, \ldots, e_{n}\right]^{T}=\left[e, \dot{e}, \ldots, e^{(n-1)}\right]^{T}$

By using the fact that $\underline{\dot{y}}_{r}=A \underline{y}_{r}+B y_{r}^{(n)}$, we get

$\dot{e}=A e+B\left[y_{r}^{(n)}-f(x)-g(x) u-d(t)\right], e_{1}=C^{T} e$.

Based on the feedback linearization approach, when the functions $f(x)$ and $g(x)$ are known, $d(t)=0$ and the state $x$ is available for measurement, the so-called ideal controller can be chosen as follows:

$u=u^{*}=g^{-1}(\underline{x})\left[-f(\underline{x})+y_{r}^{(n)}+K_{c}^{T} \underline{e}\right]$

where $K_{c}=\left[k_{c 1}, k_{c 2}, \ldots, k_{c n}\right]^{T} \in R^{n}$ is the feedback-gain vector to be selected such that the characteristic polynomial of $A-B K_{c}^{T}$ is strictly Hurwitz.

Substituting (5) into (4) yields

$e^{(n)}+K_{c}^{T} e=e^{(n)}+k_{c n} e^{(n-1)}+\cdots+k_{c 1} e=0$

Thus, it can be obtained that $\lim _{t \rightarrow \infty} e(t)=0$. However, since the functions $f(x)$ and $g(x)$ are unknown and the state vector $x$ is not available for measurement, the ideal controller (5) cannot be implemented. Thereafter, to overcome such problems, we will use:

- adaptive fuzzy systems to approximate the unknown nonlinear functions $(f(\underline{x})$ and $g(\underline{x}))$,

- an observer to estimate the tracking error vector. From the estimate of the tracking-error vector, we can directly determine the estimate of the state vector $\underline{x}$.

\section{Remark 3.}

1. Note that the system (2) is written in the observability canonical-form. This system is observable for any input: such a feature is vital for the observer design.

2. The form of the system (2) is also called the Byrnes-Isidori normal form [36]. This form is controllable, if $g(x) \neq 0$.

3. The controllability property of the pair $(A, B)$ does not imply the controllability of the nonlinear system (1) (or (2)). But, this property guarantees the existence of a feedback-gain vector, $K_{c}$, so that the characteristic polynomial of $A-B K_{c}^{T}$ is strictly Hurwitz.

4. Also, the observability property of the pair $\left(C^{T}, A\right)$ assures the existence of an observer-gain vector, $K_{0}$, so that the characteristic polynomial of $A-K_{o} C^{T}$ is strictly Hurwitz. 


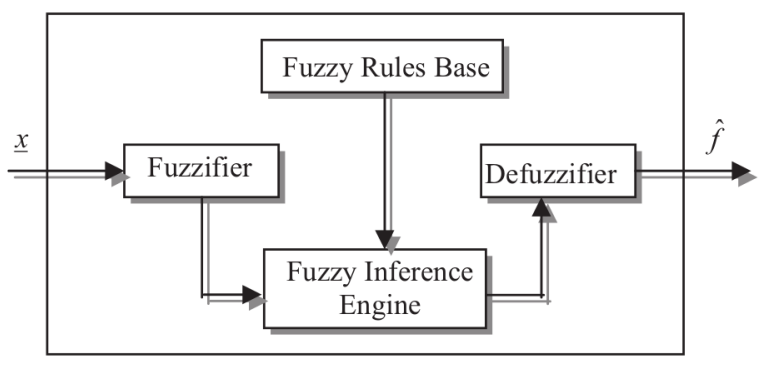

Fig. 1. The basic configuration of a fuzzy logic system.

\subsection{Description of the fuzzy logic system}

The basic configuration of a fuzzy logic system consists of a fuzzifier, some fuzzy IF-THEN rules, a fuzzy inference engine and a defuzzifier, as shown in Fig. 1.

The fuzzy inference engine uses the fuzzy IF-THEN rules to perform a mapping from an input vector $\underline{x}^{T}=\left[x_{1}, x_{2}, \ldots, x_{n}\right] \in R^{n}$ to an output $\hat{f} \in R$. The $i$ th fuzzy rule is written as

$R^{(i)}$ : if $x_{1}$ is $A_{1}^{i}$ and $\ldots$ and $x_{n}$ is $A_{n}^{i}$ then $\hat{f}$ is $f^{i}$

where $A_{1}^{i}, A_{2}^{i}, \ldots$, and $A_{n}^{i}$ are fuzzy sets and $f^{i}$ is the fuzzy singleton for the output in the $i$ th rule. By using the singleton fuzzifier, product inference, and center-average defuzzifier, the output of the fuzzy system can be expressed as follows:

$$
\begin{aligned}
\hat{f}(\underline{x}) & =\frac{\sum_{i=1}^{m} f^{i}\left(\prod_{j=1}^{n} \mu_{A_{j}^{i}}\left(x_{j}\right)\right)}{\sum_{i=1}^{m}\left(\prod_{j=1}^{n} \mu_{A_{j}^{i}}\left(x_{j}\right)\right)} \\
& =\theta^{T} \psi r(\underline{x})
\end{aligned}
$$

where $\mu_{A_{i}}\left(x_{j}\right)$ is the degree of membership of $x_{j}$ to $A_{j}^{i}, m$ is the number of fuzzy rules, $\theta^{T}=\left[f^{1}, f^{2}, \ldots, f^{m}\right]$ is the adjustable parameter vector (composed of consequent parameters), and $\psi^{T}=\left[\psi^{1} \psi^{2} \ldots \psi^{m}\right]$ with

$\psi^{i}(\underline{x})=\frac{\left(\prod_{j=1}^{n} \mu_{A_{j}^{i}}\left(x_{j}\right)\right)}{\sum_{i=1}^{m}\left(\prod_{j=1}^{n} \mu_{A_{j}^{i}}\left(x_{j}\right)\right)}$

being the fuzzy basis function (FBF). Throughout the paper, it is assumed that the FBFs are selected so that there is always at least one active rule [3], i.e. $\Sigma_{i=1}^{m}\left(\Pi_{j=1}^{n} \mu_{A_{i}^{i}}\left(x_{j}\right)>0\right)$.

It is worth noticing that the fuzzy system (7) is widely applied in modeling, identification and control of nonlinear systems because it has been proven by [3] that this simple fuzzy system can approximate an arbitrary nonlinear smooth function $f(x)$ defined on a compact operating space to an given accuracy. Of particular importance, it is assumed that the FBFs $\psi(x)$ are properly specified beforehand by designer. But, the consequent parameters $\theta$ are determined by appropriate adaptation laws.

\section{Observer-based fuzzy adaptive controller}

Consider now the following observer for estimating the tracking error vector $\underline{e}$ :

$\underline{\hat{e}}=A_{C} \underline{\hat{e}}+K_{o} \tilde{e}_{1}, \hat{e}_{1}=C^{T} \underline{\hat{e}}$.

where $A_{c}=A-B K_{c}^{T}, \quad \tilde{e}_{1}=e_{1}-\hat{e}_{1}=\hat{y}-y, \underline{\hat{e}}=\underline{y}_{r}-\underline{\hat{x}}$, with $\underline{\hat{x}}$ is the estimate of the state vector $\underline{x}$ and $\hat{e}$ is the estimate of the tracking error vector e. $K_{o}=\left[k_{01}, k_{02}, \ldots, \bar{k}_{o n}\right]^{T} \in R^{n}$ is the observer-gain vector to be selected such that the characteristic polynomial of $A-K_{0} C^{T}$ is strictly Hurwitz, and the vector $K_{c}$ has been previously defined.
Let us define the observation error vector as $\tilde{e}=$ $\left[\tilde{e}_{1}, \tilde{e}_{2}, \ldots, \tilde{e}_{n}\right]^{T}=\underline{e}-\underline{\hat{e}}$. Subtracting (8) from (4), we get the dynamics of the observation error as

$\underline{\dot{\tilde{e}}}=A_{o} \underline{\tilde{e}}+B[\hat{v}-f(\underline{x})-g(\underline{x}) u-d(t)]$,

$\tilde{e}_{1}=C^{T} \tilde{e}$.

with $A_{o}=A-K_{o} C^{T}$, where $\hat{v}=y_{r}^{(n)}+K_{c}^{T} \hat{e}$.

The unknown continuous nonlinear functions $f(\underline{x})$ and $g(\underline{x})$ can be approximated respectively, on the compact set $\Omega_{x}$, by the fuzzy system (7) as follows:

$\hat{f}\left(\underline{x}, \theta_{f}\right)=\theta_{f}^{T} \psi_{f}(\underline{x})$,

$\hat{g}\left(\underline{x}, \theta_{g}\right)=\theta_{g}^{T} \psi_{g}(\underline{x})$,

where $\psi_{f}(\underline{x})$ and $\psi_{g}(\underline{x})$ are FBF vectors fixed a priori by the designer, and $\theta_{f}$ and $\theta_{g}$ are the adjustable parameter vector of the fuzzy system.

The respective optimal values of $\theta_{f}$ and $\theta_{g}$ are defined as

$\theta_{f}^{*}=\underset{\theta_{f}}{\arg \min }\left[\sup _{x \in \Omega_{\underline{x}}}\left|f(\underline{x})-\hat{f}\left(\underline{x}, \theta_{f}\right)\right|\right]$

$\theta_{g}^{*}=\underset{\theta_{g}}{\arg \min }\left[\sup _{x \in \Omega_{\underline{x}}}\left|g(\underline{x})-\hat{g}\left(\underline{x}, \theta_{g}\right)\right|\right]$

Notice that the quantities $\theta_{f}^{*}$ and $\theta_{g}^{*}$ are introduced only for analysis purposes, and their values are not needed when implementing the controller $[37,38]$.

Define

$\tilde{\theta}_{f}=\theta_{f}-\theta_{f}^{*}$,

$\tilde{\theta}_{g}=\theta_{g}-\theta_{g}^{*}$

as the parameter estimation errors, and

$\varepsilon_{f}(\underline{x})=f(\underline{x})-\hat{f}\left(\underline{x}, \theta_{f}^{*}\right)$,

$\varepsilon_{g}(\underline{x})=g(\underline{x})-\hat{g}\left(\underline{x}, \theta_{g}^{*}\right)$

as the fuzzy approximation errors, where $\hat{f}\left(\underline{x}, \theta_{f}^{*}\right)=\theta_{f}^{* T} \psi_{f}(\underline{x})$ and $\hat{g}\left(\underline{x}, \theta_{g}^{*}\right)=\theta_{g}^{* T} \psi_{g}(\underline{x})$.

As in the literature $[3,26,39-43]$, we assume that those fuzzy approximation errors are bounded for all $\forall \underline{x} \in \Omega_{x}$, i.e.

$\left|\varepsilon_{f}(\underline{x})\right| \leq \bar{\varepsilon}_{f}$ and $\left|\varepsilon_{g}(\underline{x})\right| \leq \bar{\varepsilon}_{g}, \quad \forall x \in \Omega_{x}$

where $\bar{\varepsilon}_{f}$ and $\bar{\varepsilon}_{g}$ are unknown positive constants.

Since the state vector $x$ is not available for measurement, the fuzzy systems (10) and (11) used to approximate the unknown functions $(f(x)$ and $g(x))$ are replaced by the following fuzzy systems:

$\hat{f}\left(\underline{\hat{x}}, \theta_{f}\right)=\theta_{f}^{T} \psi_{f}(\underline{\hat{x}})$,

$\hat{g}\left(\underline{\hat{x}}, \theta_{g}\right)=\theta_{g}^{T} \psi_{g}(\underline{\hat{x}})$

where the vector $\hat{x}$ is the estimate of $x$.

From (14)-(17) and (19) and (20), we have

$$
\begin{aligned}
f(\underline{x}) & =f(\underline{x})-\hat{f}\left(\underline{x}, \theta_{f}^{*}\right)+\hat{f}\left(\underline{x}, \theta_{f}^{*}\right)-\hat{f}\left(\underline{\hat{x}}, \theta_{f}^{*}\right)+\hat{f}\left(\underline{\hat{x}}, \theta_{f}^{*}\right) \\
& =\theta_{f}^{* T} \psi_{f}(\underline{\hat{x}})+\varepsilon_{f}(\underline{x})+\theta_{f}^{* T}\left[\psi_{f}(\underline{x})-\psi_{f}(\underline{\hat{x}})\right], \\
& =\theta_{f}^{* T} \psi_{f}(\underline{\hat{x}})+w_{f}(\underline{x}, \underline{\hat{x}}) .
\end{aligned}
$$

$$
\begin{aligned}
g(\underline{x}) & =g(\underline{x})-\hat{g}\left(\underline{x}, \theta_{g}^{*}\right)+\hat{g}\left(\underline{x}, \theta_{g}^{*}\right)-\hat{g}\left(\underline{\hat{x}}, \theta_{g}^{*}\right)+\hat{g}\left(\underline{\hat{x}}, \theta_{g}^{*}\right) \\
& =\theta_{g}^{* T} \psi_{g}(\underline{\hat{x}})+\varepsilon_{g}(\underline{x})+\theta_{g}^{* T}\left[\psi_{g}(\underline{x})-\psi_{g}(\underline{\hat{x}})\right], \\
& =\theta_{g}^{* T} \psi_{g}(\underline{\hat{x}})+w_{g}(\underline{x}, \underline{\hat{x}}) .
\end{aligned}
$$


with $\quad w_{f}(\underline{x}, \underline{\hat{x}})=\varepsilon_{f}(\underline{x})+\theta_{f}^{* T}\left[\psi_{f}(\underline{x})-\psi_{f}(\underline{\hat{x}})\right] \quad$ and $\quad w_{g}(\underline{x}, \underline{\hat{x}})=\varepsilon_{g}(\underline{x})+$ $\theta_{g}^{* T}\left[\psi_{g}(\underline{x})-\psi_{g}(\underline{\hat{x}})\right]$ are the approximation errors. Notice that $w_{f}(\underline{x}, \underline{\hat{x}})$ and $w_{g}(\underline{x}, \underline{\hat{x}})$ have also upper bounds [3,26].

Substituting (21) and (22) into (9) yields

$\underline{\dot{\tilde{e}}}=A_{o} \underline{\tilde{e}}+B\left[\hat{v}-\hat{f}\left(\underline{\hat{x}}, \theta_{f}\right)-g\left(\underline{\hat{x}}, \theta_{g}\right) u+\tilde{\theta}_{f}^{T} \psi_{f}(\underline{\hat{x}})+\tilde{\theta}_{g}^{T} \psi_{g}(\underline{\hat{x}}) u-w_{1}\right]$,

$\tilde{e}_{1}=C^{T} \tilde{e}$.

where $w_{1}=w_{f}(\underline{x}, \underline{\hat{x}})+w_{g}(\underline{x}, \underline{\hat{x}}) u+d(t), \tilde{\theta}_{f}=\theta_{f}-\theta_{f}^{*}$ and $\tilde{\theta}_{g}=\theta_{g}-\theta_{g}^{*}$ are the parameter approximation errors.

The control input for the system (1) can be determined as

$u=\frac{\operatorname{Tanh}\left(\delta_{2} \theta_{g}^{T} \psi_{g}(\underline{\hat{x}})\right)}{\theta_{g}^{T} \psi_{g}(\underline{\hat{x}}) \operatorname{Tanh}\left(\delta_{2} \theta_{g}^{T} \psi_{g}(\underline{\hat{x}})\right)+\delta_{1}}\left(-\theta_{f}^{T} \psi_{f}(\underline{\hat{x}})+\operatorname{Sat}(\hat{v})+u_{r}\right)$

where $\delta_{1}$, and $\delta_{2}$ are strictly positive design constants, Tanh(.) denotes the hyperbolic tangent function and Sat(.) the usual saturation function. $u_{r}$ is a dynamic adaptive control term which will be designed later.

Substituting the control input (24) into (23) yields

$\underline{\dot{\tilde{e}}}=A_{o} \underline{\tilde{e}}+B\left[-u_{r}+\tilde{\theta}_{f}^{T} \psi_{f}(\underline{\hat{x}})+\tilde{\theta}_{g}^{T} \psi_{g}(\underline{\hat{x}}) u+w_{2}\right]$,

$\tilde{e}_{1}=C^{T} \tilde{e}$.

where

$w_{2}=-w_{1}+\bar{u}+\hat{v}-\operatorname{Sat}(\hat{v})$

and

$\bar{u}=\frac{\delta_{1}}{\theta_{g}^{T} \psi_{g}(\underline{\hat{x}}) \operatorname{Tanh}\left(\delta_{2} \theta_{g}^{T} \psi_{g}(\underline{\hat{x}})\right)+\delta_{1}}\left(-\theta_{f}^{T} \psi_{f}(\underline{\hat{x}})+\operatorname{Sat}(\hat{v})+u_{r}\right)$

Since only the output observation-error $\tilde{e}_{1}$ in (25) is measurable, one will use the SPR-Lyapunov design approach to analyze the stability of the observation error dynamics (25) and to generate the adaptive laws to estimate the fuzzy parameter vectors and unknown constants.

The dynamics (25) can be expressed in frequency domain using the mixed notation (i.e. time-frequency)

$\tilde{e}_{1}=H(s)\left[-u_{r}+\tilde{\theta}_{f}^{T} \psi_{f}(\underline{\hat{x}})+\tilde{\theta}_{g}^{T} \psi_{g}(\underline{\hat{x}}) u+w_{2}\right]$

where $s$ is the Laplace variable and $H(s)=C^{T}\left(s I-A_{o}\right)^{-1} B$ is the stable transfer function of (25). Note that this notation is very common in the adaptive control literature such as in $[32,41$, 44-47]. It also refers to the convolution between the inverse Laplace transform of $H(s)$ and the term $\left[-u_{r}+\tilde{\theta}_{f}^{T} \psi_{f}(\underline{\hat{x}})+\tilde{\theta}_{g}^{T} \psi_{g}\right.$ $\left.(\hat{x}) u+w_{2}\right]$.

Now, since $H(s)$ in (27) is not SPR in general, we introduce a low pass filter $T(s)$ such that $\bar{H}(s)=H(s) T^{-1}(s)$ is SPR:

$$
\begin{aligned}
\tilde{e}_{1}= & \bar{H}(s)\left(-u_{r f}+T(s)\left[\theta_{f}^{T} \psi_{f}(\underline{\hat{x}})+\theta_{g}^{T} \psi_{g}(\underline{\hat{x}}) u\right]-\theta_{f}^{* T}\left[T(s) \psi_{f}(\underline{\hat{x}})\right]\right. \\
& \left.-\theta_{g}^{* T}\left[T(s) \psi_{g}(\underline{\hat{x}}) u\right]+w_{2 f}\right)
\end{aligned}
$$

with $u_{r f}=T(s)\left[u_{r}\right], \quad$ and $w_{2 f}=-T(s)\left[w_{f}(\underline{x}, \underline{\hat{x}})\right]-T(s)\left[w_{g}(\underline{x}, \underline{\hat{x}}) u\right]$

$$
-T(s)[d(t)]+T(s)[u]+T(s)[\hat{v}-\operatorname{Sat}(\hat{v})]
$$

From (28), it is clear that the presence of the filtered terms $u_{r f}$ and $T(s)\left[\theta_{f}^{T} \psi_{f}(\underline{\hat{x}})+\theta_{g}^{T} \psi_{g}(\underline{\hat{x}}) u\right]$ in the output observation-error dynamics makes the control system design very difficult.

Remark 4. $H(s)$ is SPR, with $s=\sigma+j \omega$, if the following three conditions are satisfied [48]:

(a) When $s$ is real, $H(s)$ is real.

(b) The poles of $H(s)$ are not in the right half plane.

(c) For any real $\omega$, the real part of $H(j \omega)$ is positive, i.e. $\operatorname{Re}[H(j \omega)] \geq 0$.
Remark 5. For example, one can easily show that for $n=2, H(s)$ is not SPR (because the real part of $H(j \omega)$ can be negative, i.e. the third condition in Remark 4 is not satisfied). One will note this effect in the simulation examples given in Section 4.

Let us define a novel error $e_{m 1}$, called the modified error, as follows:

$e_{m 1}=\tilde{e}_{1}+e_{a 1}$

where the error $e_{a 1}$ is called the auxiliary error, which is generated by the following dynamics:

$e_{a 1}=\bar{H}(s)\left(u_{r f}-u_{r}-T(s)\left[\theta_{f}^{T} \psi_{f}(\underline{\hat{x}})+\theta_{g}^{T} \psi_{g}(\underline{\hat{x}}) u\right]+\theta_{f}^{T} \psi_{f}(\underline{\hat{x}})+\theta_{g}^{T} \psi_{g}(\underline{\hat{x}}) u\right)$

From (28), (30) and (31), the dynamics of the modified error $e_{m 1}$ can be expressed as follows:

$e_{m 1}=\bar{H}(s)\left(-u_{r}+\tilde{\theta}_{f}^{T} \psi_{f}(\underline{\hat{x}})+\tilde{\theta}_{g}^{T} \psi_{g}(\underline{\hat{x}}) u+w_{3}\right)$

where

$w_{3}=w_{2 f}-\theta_{f}^{* T}\left[T(S) \psi_{f}(\underline{\hat{x}})\right]-\theta_{g}^{* T}\left[T(S) \psi_{g}(\underline{\hat{x}}) u\right]+\theta_{f}^{* T} \psi_{f}(\underline{\hat{x}})+\theta_{g}^{* T} \psi_{g}(\underline{\hat{x}}) u$.

Now, to facilitate the controller design and the stability analysis, we make the following mild assumption:

Assumption 4. The following inequality holds

$\left|w_{3}\right| \leq a_{0}+a_{1}\left|\bar{u}_{f}\right|+a_{2}\left|u_{f}\right|+a_{3}|u|+a_{4}\left|\Delta \hat{v}_{f}\right|=\kappa^{* T} \varphi$

where $\kappa^{*}=\left[a_{0}, a_{1}, a_{2}, a_{3}, a_{4}\right]^{T}$ is an unknown positive constant vector, $\quad \bar{u}_{f}=T(s)[\bar{u}], \quad u_{f}=T(s)[u], \quad \Delta \hat{v}_{f}=T(s)[\hat{v}-\operatorname{Sat}(\hat{v})] \quad$ and $\varphi=\left[1,\left|\bar{u}_{f}\right|,\left|u_{f}\right|,|u|,\left|\Delta \hat{v}_{f}\right|\right]^{T}$.

The state space realization of (33) is given by:

$\underline{\dot{e}}_{m}=\bar{A}_{o} \underline{e}_{m}+\bar{B}\left[-u_{r}+\tilde{\theta}_{f}^{T} \psi_{f}(\underline{\hat{x}})+\tilde{\theta}_{g}^{T} \psi_{g}(\underline{\hat{x}}) u+w_{3}\right]$,

$e_{m 1}=\bar{C}^{T} \underline{e}_{m}$,

where $e_{m}=\left[e_{m 1}, e_{m 2}, \ldots, e_{m n}\right]^{T}$ and $\left(\bar{A}_{o} \in R^{n \times n}, \bar{B} \in R^{n}, \bar{C} \in R^{n}\right)$ is a minimal state realization of $\bar{H}(s)=H(s) T^{-1}(s)=\bar{C}^{T}\left(s I-\bar{A}_{0}\right)^{-1} \bar{B}$, with $\bar{C}=[1,0, \ldots, 0]^{T}$.

Since $\bar{H}(s)$ is SPR, the following holds:

$\bar{A}_{0}^{T} P+P \bar{A}_{0}=-Q<0$

$P \bar{B}=\bar{C}$

where $P=P^{T}>0$ and $Q=Q^{T}>0$. Note that the matrix Eq. (35) and the dynamics (34) will be used later in the stability analysis.

In order to dynamically compensate for the uncertain term $w_{3}$, the robust control term $u_{r}$ can be designed as follows:

$\dot{u}_{r}=-\gamma_{r} u_{r}+\gamma_{r}\left[e_{m 1}-\frac{\operatorname{Tanh}\left(\delta_{3} u_{r}\right)}{u_{r} \operatorname{Tanh}\left(\delta_{3} u_{r}\right)+\delta_{4}^{2}} \kappa^{T} \varphi\left|e_{m 1}\right|\right]$,

with

$\dot{\delta}_{4}=-\gamma_{\delta} \sigma_{\delta} \delta_{4}-\gamma_{\delta} \frac{\delta_{4}}{u_{r} \operatorname{Tanh}\left(\delta_{3} u_{r}\right)+\delta_{4}^{2}} \kappa^{T} \varphi\left|e_{m 1}\right| \quad$ with $\delta_{4}(0)>0$

$\dot{\kappa}=-\gamma_{\kappa} \sigma_{\kappa} \kappa+\gamma_{\kappa}\left|e_{m 1}\right| \varphi \quad$ with $\kappa_{i}(0) \geq 0$

where $\gamma_{r}, \gamma_{\delta}, \gamma_{k}, \sigma_{\kappa}, \sigma_{\delta}$ and $\delta_{3}$ are positive design constants, $\kappa \in R^{5}$ is the estimate of the unknown vector $\kappa^{*}$.

The adaptation laws for $\theta_{f}$ and $\theta_{g}$ can be determined as follows:

$\dot{\theta}_{f}=-\gamma_{f} \sigma_{f} \theta_{f}-\gamma_{f} e_{m 1} \psi_{f}(\underline{\hat{x}})$

$\dot{\theta}_{g}=-\gamma_{g} \sigma_{g} \theta_{g}-\gamma_{g} e_{m 1} u \psi_{g}(\underline{\hat{x}})$

where $\gamma_{f}, \gamma_{g}, \sigma_{f}$ and $\sigma_{g}$ are positive design constants.

Fig. 2 shows the scheme of the proposed fuzzy indirect adaptive controller. 


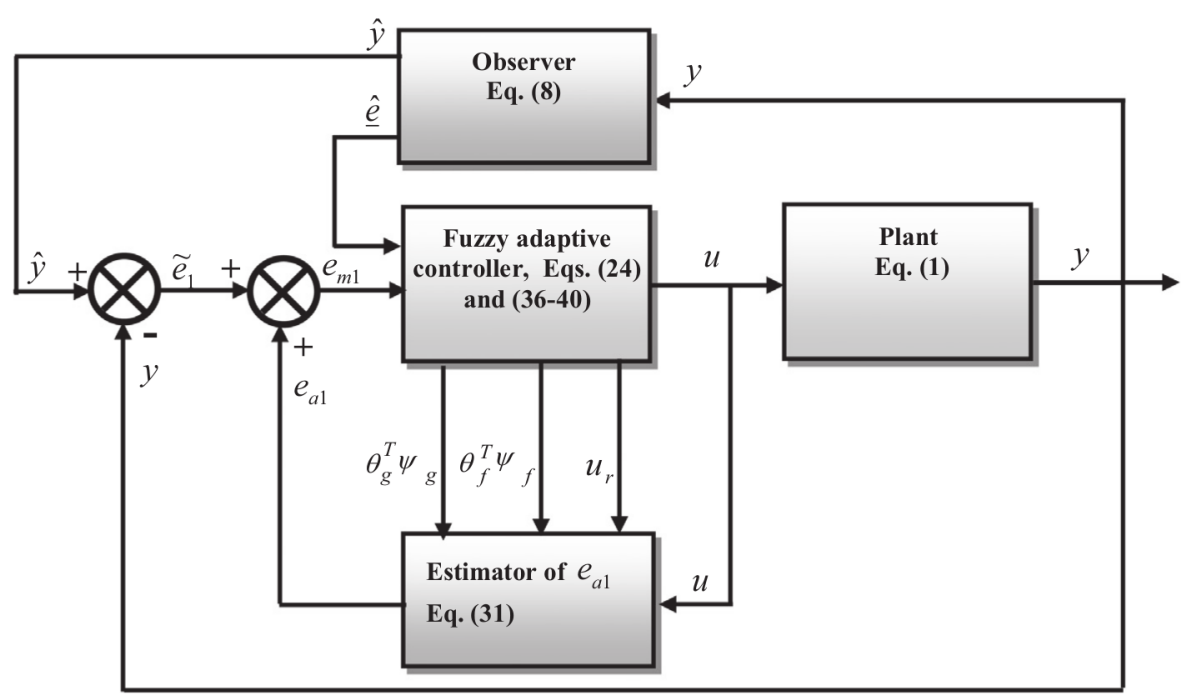

Fig. 2. The scheme of the proposed fuzzy indirect adaptive controller based on observer.

The following theorem establishes the stability and performance properties of the closed-loop system with this proposed controller.

Theorem 1. Consider the system (1) under Assumptions 1-4 and the observer (8). Then, the proposed fuzzy adaptive controller, defined by (24), (30), (31) and (36)-(40), guarantees the following nice properties:

1. All signals in the closed-loop system are bounded, i.e. $\underline{e}_{m}, e_{m 1}, e_{a 1}, \tilde{e}_{1}, \underline{\tilde{e}}, \underline{\hat{e}}, \underline{e}, \theta_{f}, \theta_{g} u_{r}, \delta_{4}, \kappa$ and $u \in L_{\infty}$,

2. The output tracking error converges to the residual set $\Omega_{e}=\left\{e_{1}|\quad| e_{1} \mid \leq \eta_{e}\right.$, where $\eta_{e}$ is a positive constant which will be defined later.

Proof of Theorem 1. Let us consider the following Lyapunov-like function:

$V_{1}=\frac{1}{2} \underline{e}_{m}^{T} P \underline{e}_{m}+\frac{1}{2 \gamma_{f}} \tilde{\theta}_{f}^{T} \tilde{\theta}_{f}+\frac{1}{2 \gamma_{g}} \tilde{\theta}_{g}^{T} \tilde{\theta}_{g}+\frac{1}{2 \gamma_{r}} u_{r}^{2}+\frac{1}{2 \gamma_{\delta}} \delta_{4}^{2}+\frac{1}{2 \gamma_{\kappa}} \tilde{\kappa}^{T} \tilde{\kappa}$

where $\tilde{\kappa}=\kappa-\kappa^{*}$.

The time derivative of $V_{1}$ is

$\dot{V}_{1}=\frac{1}{2} \underline{e}_{m}^{T} P \underline{e}_{m}+\frac{1}{2} \underline{e}_{m}^{T} P \underline{e}_{m}+\frac{1}{\gamma_{f}} \tilde{\theta}_{f}^{T} \dot{\theta}_{f}+\frac{1}{\gamma_{g}} \tilde{\theta}_{g}^{T} \dot{\theta}_{g}+\frac{1}{\gamma_{r}} u_{r} \dot{u}_{r}+\frac{1}{\gamma_{\delta}} \delta_{4} \dot{\delta}_{4}+\frac{1}{\gamma_{\kappa}} \tilde{\kappa}^{T} \dot{\kappa}$

Evaluating (42) along the trajectories (34) and (36)-(40) gives

$\dot{V}_{1} \leq-\frac{1}{2} \underline{e}_{m}^{T} Q \underline{e}_{m}-u_{r}^{2}-\sigma_{\delta} \delta_{4}^{2}-\sigma_{f} \tilde{\theta}_{f}^{T} \theta_{f}-\sigma_{g} \tilde{\theta}_{g}^{T} \theta_{g}-\sigma_{\kappa} \tilde{\kappa}^{T} \kappa$

Since the following inequalities are valid:

$-\sigma_{f} \tilde{\theta}_{f}^{T} \theta_{f} \leq-\frac{\sigma_{f}}{2}\left\|\tilde{\theta}_{f}\right\|^{2}+\frac{\sigma_{f}}{2}\left\|\theta_{f}^{*}\right\|^{2}$,

$-\sigma_{g} \tilde{\theta}_{g}^{T} \theta_{g} \leq-\frac{\sigma_{g}}{2}\left\|\tilde{\theta}_{g} \mid\right\|^{2}+\frac{\sigma_{g}}{2}\left\|\theta_{g}^{*}\right\|^{2}$, and

$-\sigma_{\kappa} \tilde{\kappa}^{T} \kappa \leq-\frac{\sigma_{\kappa}}{2}|| \tilde{\kappa}\left\|^{2}+\frac{\sigma_{\kappa}}{2}\right\| \kappa^{*} \|^{2}$,

we can rewrite (43) as follows:

$\dot{V}_{1} \leq-\frac{1}{2} \underline{e}_{m}^{T} Q \underline{e}_{m}-u_{r}^{2}-\sigma_{\delta} \delta_{4}^{2}-\frac{\sigma_{f}}{2}\left\|\tilde{\theta}_{f}\right\|^{2}-\frac{\sigma_{g}}{2}\left\|\tilde{\theta}_{g}\right\|^{2}-\frac{\sigma_{\kappa}}{2}\|\tilde{\kappa}\|^{2}+\pi_{1}$ where

$\pi_{1}=\frac{\sigma_{f}}{2}\left\|\theta_{f}^{*}\right\|^{2}+\frac{\sigma_{g}}{2}\left\|\theta_{g}^{*}\right\|^{2}+\frac{\sigma_{\kappa}}{2}\left\|\kappa^{*}\right\|^{2}$.

Let $\mu_{1}=\min \left\{\lambda_{\min }(Q) / \lambda_{\max }(P), 2 \gamma_{r}, 2 \gamma_{\delta} \sigma_{\delta}, \gamma_{f} \sigma_{f}, \gamma_{g} \sigma_{g}, \gamma_{\kappa} \sigma_{\kappa}\right\}$, hence we can rewrite (44) as follows:

$\dot{V}_{1} \leq-\mu_{1} V_{1}+\pi_{1}$

where $\lambda_{\min }(Q)$ denotes the smallest eigen-value of $Q$, and $\lambda_{\max }(P)$ the largest eigen-value of $P$.

Multiplying (45) by $e^{\mu_{1} t}$ leads to the following result:

$\frac{d}{d t}\left(V_{1} e^{\mu_{1} t}\right) \leq \pi_{1} e^{\mu_{1} t}$

Integrating (46) over $[0, t]$ yields

$0 \leq V_{1}(t) \leq \frac{\pi_{1}}{\mu_{1}}+\left(V_{1}(0)-\frac{\pi_{1}}{\mu_{1}}\right) e^{-\mu_{1} t}$

From (47), therefore, the modified error $\underline{e}_{m}$, the robust term $u_{r}$, the adaptive parameter $\delta_{4}$ and the parameter errors $\left(\tilde{\theta}_{f}, \tilde{\theta}_{g}\right.$ and $\left.\tilde{\kappa}\right)$ are UUB.

With the help of $(41), V_{1}(0)$ is defined as follows:

$$
\begin{aligned}
V_{1}(0)= & \frac{1}{2} \underline{e}_{m}^{T}(0) P \underline{e}_{m}(0)+\frac{1}{2 \gamma_{f}} \tilde{\theta}_{f}^{T}(0) \tilde{\theta}_{f}(0)+\frac{1}{2 \gamma_{g}} \tilde{\theta}_{g}^{T}(0) \tilde{\theta}_{g}(0) \\
& +\frac{1}{2 \gamma_{r}} u_{r}^{2}(0)+\frac{1}{2 \gamma_{\delta}} \delta_{4}^{2}(0)+\frac{1}{2 \gamma_{\kappa}} \tilde{\kappa}^{T}(0) \tilde{\kappa}(0)
\end{aligned}
$$

From (41) and (47), one has

$\left\|\underline{e}_{m}\right\| \leq\left(\frac{2}{\lambda_{\min }(P)}\left(\frac{\pi_{1}}{\mu_{1}}+\left(V_{1}(0)-\frac{\pi_{1}}{\mu_{1}}\right) e^{-\mu_{1} t}\right)\right)^{1 / 2}$.

Then, the solution $e_{m}$ exponentially converges to a bounded region $\Omega_{e_{m}}=\left\{\underline{e}_{m} \mid \quad\left\|\underline{e}_{m}\right\| \leq \eta_{e m}\right\}$, with

$\eta_{e m}=\left(\frac{2}{\lambda_{\min }(P)} \frac{\pi_{1}}{\mu_{1}}\right)^{1 / 2}$.

From the boundedness of $\tilde{\theta}_{f}, \tilde{\theta}_{g}$ and $\tilde{\kappa}$, one can directly conclude about the boundedness of $\theta_{f}, \theta_{g}$ and $\kappa$. The boundedness of the control $u$ follows that of $u_{r}, \theta_{f}, \theta_{g}$ and $\operatorname{Sat}(\hat{v})$. From (31), since the term $\quad\left(u_{r f}-u_{r}-L(s)\left[\theta_{f}^{T} \psi_{f}(\underline{\hat{x}})+\theta_{g}^{T} \psi_{g}(\underline{\hat{x}}) u\right]+\theta_{f}^{T} \psi_{f}(\underline{\hat{x}})+\theta_{g}^{T} \psi_{g}(\underline{\hat{x}}) u\right) \quad$ is bounded and $\bar{H}(s)$ is a stable transfer function, one can easily show that the auxiliary error $e_{a 1}$ is also bounded (i.e. it has an upper bound). Now, in order to quantifier this upper bound, one 
Table 1

Comparison between our control scheme and the previous works.

\begin{tabular}{|c|c|c|}
\hline Comparaison & Features of the proposed controller & Advantages/disadvantages \\
\hline $\begin{array}{l}\text { Scheme in } \\
{[18]}\end{array}$ & $\begin{array}{l}\text { - An indirect adaptive control has been proposed. } \\
\text { - A linear observer has been used to estimate the tracking error } \\
\text { vector. } \\
\text { - A projection algorithm has been incorporated in the adaptive } \\
\text { laws to avoid the parameters drift. } \\
\text { - The problem of an possible singularity of the controller has } \\
\text { been solved by modifying this projection algorithm. } \\
\text { - This scheme requires SPR condition on the observation error } \\
\text { dynamics } \\
\text { - A sliding mode control term has been used to deal with the } \\
\text { fuzzy approximation errors. }\end{array}$ & $\begin{array}{l}\text { Advantages } \\
\text { - The tracking errors can theoretically converge to the origin. } \\
\text { Disadvantages } \\
\text { - The filtering of the FBFs can make the dynamic order of the controller-observer } \\
\text { system very large. } \\
\text { - Authors have designed a robust control term filtered } v_{f} \text { instead of } v \text {. The } \\
\text { problem is how to obtain } v \text { from } v_{f} \text { ? } \\
\text { - The robust control term designed } v_{f} \text { is not smooth. } \\
\text { - The projection algorithm used requires the knowledge of the norm bound of } \\
\text { the uncertain optimal fuzzy parameters. } \\
\text { - The stability analysis is questionable, according to [26]. }\end{array}$ \\
\hline $\begin{array}{l}\text { Scheme in } \\
\text { [19] }\end{array}$ & $\begin{array}{l}\text { - A direct adaptive control has been designed. } \\
\text { - A linear observer has been used to estimate the tracking error } \\
\text { vector. } \\
\text { - A projection algorithm has been incorporated in the adaptive } \\
\text { laws to avoid the parameters drift. } \\
\text { - This scheme requires SPR condition on the observation error } \\
\text { dynamics } \\
\text { - A sliding mode control term has been used to compensate for } \\
\text { the fuzzy approximation errors. }\end{array}$ & $\begin{array}{l}\text { Advantages } \\
\text { - The tracking errors can theoretically converge to the origin. } \\
\text { Disadvantages } \\
\text { - Authors have designed a robust control term filtered } v_{f} \text { instead of } v \text {. The } \\
\text { problem is how to obtain } v \text { from } v_{f} \text { ? } \\
\text { - The robust control term designed } v_{f} \text { is not smooth. } \\
\text { - The projection algorithm used requires the knowledge of the norm bound of } \\
\text { the uncertain optimal fuzzy parameters. }\end{array}$ \\
\hline $\begin{array}{l}\text { Scheme in } \\
{[21]}\end{array}$ & $\begin{array}{l}\text { - Indirect and direct adaptive controllers have been designed. } \\
\text { - A linear observer has been used to estimate the tracking error } \\
\text { vector. } \\
\text { - The problem of a possible drift of the adaptive fuzzy parameters } \\
\text { has not been treated. } \\
\text { - The singularity problem of the controller has not been } \\
\text { discussed. } \\
\text { - This scheme requires SPR condition on the observation error } \\
\text { dynamics. } \\
\text { - An } H_{\infty} \text { robust control term has been used to compensate for the } \\
\text { fuzzy approximation errors. }\end{array}$ & $\begin{array}{l}\text { Advantages: } \\
\text { - The controller designed is very simple. } \\
\text { - There are fewer parameters to be adapted. } \\
\text { Disadvantages: } \\
\text { - Authors have designed the filtered control terms }\left(u_{a 1} \text { and } u_{s 1}\right) \text { instead of }\left(u_{a} \text { and }\right. \\
\left.u_{s}\right) \text {. The question is how to obtain the control terms }\left(u_{a} \text { and } u_{s}\right) \text { from }\left(u_{a 1} \text { and }\right. \\
\left.u_{s 1}\right) \text {, respectively? } \\
\text { - Because the authors have not treated the singularity problem and parameters } \\
\text { drift, they assumed that the adjusted parameters never reach some known } \\
\text { boundaries. Note that this assumption is not realistic in the practice. } \\
\text { - The FBF filtering makes the dynamic order of the controller-observer system } \\
\text { very large. } \\
\text { - Moreover, the stability analysis is questionable, according to [26]. }\end{array}$ \\
\hline $\begin{array}{l}\text { Scheme in } \\
{[26]}\end{array}$ & $\begin{array}{l}\text { - A direct adaptive control has been designed. } \\
\text { - An unified observer has been used to estimate the tracking } \\
\text { error vector. } \\
\text { - To avoid the parameters drift, a } \sigma \text {-modification term has been } \\
\text { incorporated in the adaptive laws. } \\
\text { - it does not require the SPR condition on the observation error } \\
\text { dynamics. } \\
\text { - A sliding mode term has been used to compensate for the fuzzy } \\
\text { approximation errors. }\end{array}$ & $\begin{array}{l}\text { Advantages: } \\
\text { - An unified design frame-work for the high-gain observers has been proposed. } \\
\text { - This scheme does not require the SPR condition. } \\
\text { - The FBF filtering is no longer required. } \\
\text { Disadvantages: } \\
\text { - The robust control term designed is not smooth. } \\
\text { - The tracking errors cannot converge to zero. They are only UUB. } \\
\text { - High-gain observers proposed are very sensitive to noise. }\end{array}$ \\
\hline $\begin{array}{l}\text { Our } \\
\text { proposed } \\
\text { scheme }\end{array}$ & $\begin{array}{l}\text { - An indirect adaptive control has been designed. } \\
\text { - An linear observer has been used to estimate the tracking error } \\
\text { vector. } \\
\text { - To avoid the parameters drift, a } \sigma \text {-modification term has been } \\
\text { incorporated in the adaptive laws. } \\
\text { - This scheme requires the SPR condition on the observation error } \\
\text { dynamics. } \\
\text { - A dynamic adaptive compensator has been used to compensate } \\
\text { for the fuzzy approximation errors together with other } \\
\text { uncertainties }\end{array}$ & $\begin{array}{l}\text { Advantages: } \\
\text { - Unlike in [18,21], there is no filtering of the FBF vectors. } \\
\text { - Unlike in }[18,19,21] \text {, by using the SPR condition and the Lyapunov theory, the } \\
\text { stability of the closed-loop is rigorously proven. } \\
\text { - The dynamic adaptive compensator designed is smooth. } \\
\text { - Unlike [26], the proposed observer is not an high-gain observer. } \\
\text { Disadvantages: } \\
\text { - The tracking errors cannot converge to zero. They are only UUB. }\end{array}$ \\
\hline
\end{tabular}

makes the state space realization of the dynamics (31):

$\underline{\dot{e}}_{a}=\bar{A}_{o} \underline{e}_{a}+\bar{B}\left[\bar{u}_{a}\right]$,

$e_{a 1}=\bar{C}^{T} \underline{e}_{a}$,

with

$\bar{u}_{a}=u_{r f}-u_{r}-L(s)\left[\theta_{f}^{T} \psi_{f}(\underline{\hat{x}})+\theta_{g}^{T} \psi_{g}(\underline{\hat{x}}) u\right]+\theta_{f}^{T} \psi_{f}(\underline{\hat{x}})+\theta_{g}^{T} \psi_{g}(\underline{x}) u$,

where $\underline{e}_{a}=\left[e_{a 1}, e_{a 2}, \ldots, e_{a n}\right]^{T}$ and $\left(\bar{A}_{0} \in R^{n \times n}, \bar{B} \in R^{n}, \bar{C} \in R^{n}\right)$ is a minimal state realization of $\bar{H}(s)=H(s) T^{-1}(s)=\bar{C}^{T}\left(s I-\bar{A}_{o}\right)^{-1} \bar{B}$ with $\bar{C}=[1,0, \ldots, 0]^{T}$.
Let us consider the following Lyapunov-like function:

$V_{2}=\frac{1}{2} e_{a}^{T} P \underline{e}_{a}$

Its time derivative is given by

$$
\begin{aligned}
\dot{V}_{2} & =\frac{1}{2} \underline{e}_{a}^{T} P \dot{e}_{a}+\frac{1}{2} \dot{e}_{a}^{T} P \underline{e}_{a} \\
& =-\frac{1}{2} \underline{e}_{a}^{T} Q \underline{e}_{a}+\underline{e}_{a}^{T} P \bar{B} \bar{u}_{a} \\
& \leq-\frac{1}{2} \lambda_{\min }(Q)\left\|\underline{e}_{a}\right\|^{2}+\frac{c_{1}}{2 \varepsilon}\left\|\underline{e}_{a}\right\|^{2}+\frac{\varepsilon}{2}
\end{aligned}
$$




$$
\leq-\mu_{2} V_{2}+\pi_{2}
$$

with $c_{1}=\| P \bar{B}||^{2}\left|\bar{u}_{a}\right|^{2}, \mu_{2}=\left(\lambda_{\min }(Q)-\left(c_{1} / \varepsilon\right)\right) / \lambda_{\max }(P)$ and $\pi_{2}=\varepsilon / 2$, where $\varepsilon$ is a small positive constant, and $\lambda_{\min }(Q)>\left(c_{1} / \varepsilon\right)$.

From (52) and (53), one has

$\left\|\underline{e}_{a}\right\| \leq\left(\frac{2}{\lambda_{\min }(P)}\left(\frac{\pi_{2}}{\mu_{2}}+\left(V_{2}(0)-\frac{\pi_{2}}{\mu_{2}}\right) e^{-\mu_{2} t}\right)\right)^{1 / 2}$.

Then, the solution $\underline{e}_{a}$ exponentially converges to a bounded region $\Omega_{e_{a}}=\left\{\underline{e}_{a} \mid \quad\left\|\underline{e}_{a}\right\| \leq \eta_{e a}\right\}$, with

$\eta_{e a}=\left(\frac{2}{\lambda_{\min }(P)} \frac{\pi_{2}}{\mu_{2}}\right)^{1 / 2}$.

From (30) and the bounds of the errors $\underline{e}_{a}$ and $\underline{e}_{m}$, one can get the upper bound of the output tracking error $e_{1}$ as follows:

$\left|\tilde{e}_{1}\right| \leq\left|e_{m 1}\right|+\left|e_{a 1}\right| \leq \eta_{\tilde{e}}$

where

$\eta_{\tilde{e}}=\left(\frac{2}{\lambda_{\min }(P)} \frac{\pi_{2}}{\mu_{2}}\right)^{1 / 2}+\left(\frac{2}{\lambda_{\min }(P)} \frac{\pi_{1}}{\mu_{1}}\right)^{1 / 2}$

From the observer dynamics (8), one can demonstrate that $\hat{e}_{1}$ and $\hat{e}$ are bounded and converge to a bounded adjustable region.

From (55) and because $\hat{e}_{1}$ and $\hat{e}$ are bounded, then the output tracking error remains in a compact set $\Omega_{e}$ specified as: $\Omega_{e}=\left\{e_{1}|\quad| e_{1} \mid \leq \eta_{e}\right\}$ where $\eta_{e}=\eta_{\tilde{e}}+\eta_{\hat{e}}$ with $\eta_{\hat{e}} \geq\left\|\hat{e}_{1}\right\|$. This ends the proof. $\square$

Remark 6. This paper considers only single-input single-output (SISO) systems to simplify the representation. The extension of our fundamental results to the following class of multivariable nonlinear uncertain systems is straightforward (that is why we omitted the control design for this class of multivariable systems):

$\dot{x}_{p 1}=x_{p 2}$

$\dot{x}_{p 2}=x_{p 3}$

$\dot{x}_{p n_{p}}=f_{p}\left(x_{1}, \ldots, x_{m}\right)+g_{p}\left(x_{1}, \ldots, x_{m}\right) u_{p}+d(t)$ for $p=1,2, \ldots, m$.

where $f_{p}\left(x_{1}, \ldots, x_{m}\right)$ is the unknown nonlinear functions of the $p$ th subsystem, $u_{p}$ is the input of the $p$ th subsystem, $g_{p}\left(x_{1}, \ldots, x_{m}\right) \neq 0$ is the unknown positive control gain. $x=\left[x_{1}^{T}, \ldots, x_{m}^{T}\right]^{T}$ is the overall state vector, and $x_{1}=\left[x_{p 1}, \ldots, x_{p n_{p}}\right]^{T}$ is the state vector of the $p$ th subsystem. Note that the model (56) can be used to describe a relatively large class of multivariable nonlinear dynamical systems, namely: the robotic manipulator used in [49], the induction motor [50], the mass-spring-damper system in [51], the unified chaotic systems [52], and many others.

Remark 7. Note that some closely related works have been investigated in $[18,19,21,26]$. Unlike in [26], the stability of the closed-loop system has been proven in $[8,9,11]$ and in this paper by using the SPR condition and the Lyapunov theory. A detailed comparison between our present work and that of $[18,19,21,26]$ is summarized in Table 1.

\section{Simulation results}

Simulation studies are carried out to show the effectiveness of the proposed controller. Two control problems are considered at this end. The first one concerns a Duffing oscillator, while the second one concerns an inverted pendulum system.

\subsection{Example 1}

In the following, we present simulation results showing the performances of the proposed controller applied to a Duffing oscillator. This chaotic system can be described as [53]

$\left\{\begin{array}{l}\dot{x}_{1}=x_{2} \\ \dot{x}_{2}=-p_{1} x_{2}-p_{2} x_{1}-p_{3} x_{1}^{3}+q \cos (\omega t)\end{array}\right.$

where $\underline{x}=\left[x_{1}, x_{2}\right]^{T}=[x, \dot{x}]^{T}$ is the state vector, $p_{1}, p_{2}, p_{3}$, and $q$ are positive constants, $t$ is the time variable, and $\omega$ is the frequency. Depending on the choice of these constants, it is known that the solutions of (57) exhibit periodic, almost periodic, and chaotic behavior [53]. A typical chaotic behavior of the uncontrolled Duffing equation can be obtained with

$p_{1}=0.4, p_{2}=-1.1, p_{3}=1, q=2.1$ and $\omega=1.8$

Then, the controlled Duffing equation can be written as follows:

$\dot{x}=\left[\begin{array}{ll}0 & 1 \\ 0 & 0\end{array}\right] \underline{x}+\left[\begin{array}{l}0 \\ 1\end{array}\right](f(\underline{x})+g(\underline{x}) u+d(t))$

$y=\left[\begin{array}{ll}1 & 0\end{array}\right] \underline{x}$

where $f(x)=-p_{1} x_{2}-p_{2} x_{1}-p_{3} x_{1}^{3}+q \cos (\omega t)$ and $g(x)=1$. The external disturbance $d(t)$ is selected as a square wave having an amplitude \pm 1 with a period of $2 \pi(s)$.

The control objective is to force the system output $y$ to track the reference signal $y_{r}=\sin (t)$. It is worth noting that the function $f(x)$ and the control gain $g(x)$ are assumed here to be unknown by the controller. In addition, the state vector is assumed to be nonmeasurable and only the system output $y$ is measurable. In fact, the model (58) is only required for simulation purposes.

The observer-gain vector and the feedback-gain vector are selected respectively as follows: $K_{0}=[160,6400]^{T}$ and $K_{c}^{T}=[144,24]$. The transfect function $H(s)$ is given by

$H(s)=\frac{1}{s^{2}+160 s+6400}$.

According to Remark 4, $H(s)$ is not SPR because the real part of $H(j \omega)$ can be negative. One should choose a low-pass filter $T(s)$ so that $\bar{H}(s)=H(s) T^{-1}(s)=\left(1 / s^{2}+160 s+6400\right) T^{-1}(s)$ is SPR. This filter can be selected as follows:

$T(s)=\frac{1}{0.0255 s+0.8197}$

From the expression of $\bar{H}(s)$, we can find that

$\bar{A}=\left[\begin{array}{cc}-160 & 1 \\ -6400 & 0\end{array}\right], \bar{B}^{T}=\left[\begin{array}{ll}0.0255 & 0.8197\end{array}\right]$, and $\bar{C}^{T}=\left[\begin{array}{ll}1 & 0\end{array}\right]$.

Given

$Q=\left[\begin{array}{cc}10 & -5 \\ -5 & 10\end{array}\right]$

solving the matrix equation (35), one obtains the following symmetric positive-definite matrix:

$P=\left[\begin{array}{rc}200.0312 & -5.0000 \\ -5.0000 & 0.1555\end{array}\right]$

The design parameters are selected as $\gamma_{f}=10^{3}, \gamma_{g}=0.5$, $\gamma_{k}=500, \gamma_{\delta}=10^{-5}, \gamma_{r}=2, \sigma_{f}=5 \times 10^{-3}, \sigma_{g}=10^{-3}, \sigma_{k}=10^{-3}$, $\sigma_{\delta}=10^{-7}, \delta_{1}=10^{-2}, \delta_{2}=1$ and $\delta_{3}=5$.

The fuzzy membership functions are defined for the variables $\hat{x}_{1}, \hat{x}_{2}$ as follows:

$\mu_{A_{j}^{1}}\left(\hat{x}_{j}\right)=\frac{1}{2}\left(1+\operatorname{Tanh}\left(-\frac{\hat{x}_{j}+0.5}{0.4}\right)\right)$,

$\mu_{A_{j}^{2}}\left(\hat{x}_{j}\right)=\exp \left(-\frac{1}{2}\left(\frac{\hat{x}_{j}^{2}}{0.6}\right)\right), \quad$ and 

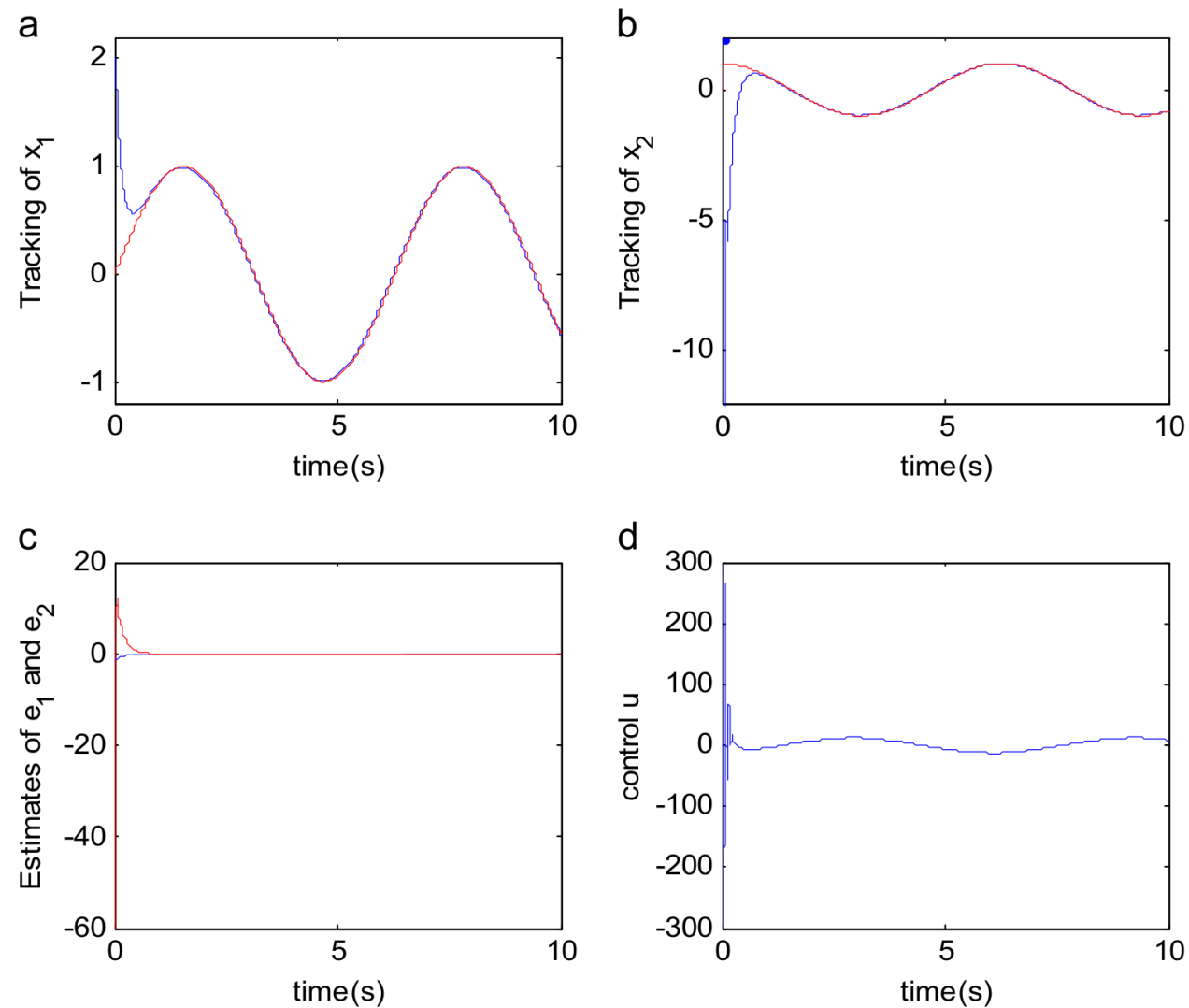

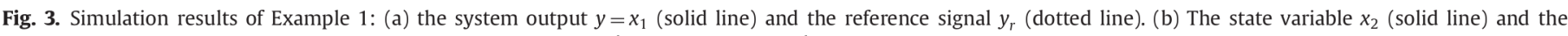
reference signal $\dot{y}_{r}$ (dotted line). (c) The tracking-errors estimates $\hat{e}_{1}$ (dotted line) and $\hat{e}_{2}$ (solid line). (d) The control signal $u$.

Table 2

IAE, ISE and IAU criteria for this proposed method and that of [26] (for Example 1).

\begin{tabular}{lllc}
\hline Control method & IAE & ISE & IAU \\
\hline $\begin{array}{l}\text { The proposed fuzzy control method } \\
\text { The fuzzy control method of [26] }\end{array}$ & $\begin{array}{l}0.5271 \\
2.02\end{array}$ & $\begin{array}{l}0.3893 \\
2.028\end{array}$ & $\begin{array}{c}107.1 \\
81.95\end{array}$ \\
\hline
\end{tabular}

$\mu_{A_{j}^{3}}\left(\hat{x}_{j}\right)=\frac{1}{2}\left(1+\operatorname{Tanh}\left(\frac{\hat{x}_{j}-0.5}{0.4}\right)\right)$

The initial conditions are chosen as $x(0)=\left[x_{1}(0), x_{2}(0)\right]^{T}=[2,2]^{T}$, $\hat{e}(0)=\left[\hat{e}_{1}(0), \hat{e}_{2}(0)\right]^{T}=[0,1]^{T}, \quad \delta_{4}(0)=1.2$ and $\kappa(0)=\left[\begin{array}{lll}0, & 0, & 0\end{array}\right.$ $\overline{0}, 0]^{T}$. The elements of $\theta_{f}(0)$ are randomly selected in the interval $\left[\begin{array}{ll}-1,1 \\ 1\end{array}\right.$. As well, the elements of $\theta_{g}(0)$ are randomly selected in the interval $[0.4,1.2]$.

Fig. 3 shows the simulation results obtained by applying the proposed fuzzy indirect adaptive controller. Fig. 3(a) and (b) illustrates the tracking performances of the state variables. The estimates $\left(\hat{e}_{1}\right.$ and $\left.\hat{e}_{2}\right)$ of tracking errors are given in Fig. 3(c). Fig. 3(d) shows the boundedness of the control signal $u$.

The fuzzy adaptive control method proposed in [26] is also simulated and compared to represent the efficiency of our method proposed in this paper. And to represent a qualitative comparison between these two controllers, integral of absolute (output-tracking) error (IAE) integral of squared error (ISE) and Integral Absolute input (IAU) criteria are calculated for each one in Table 2.

From Table 2, it is clear that the output-tracking performances (IAE and ISE) obtained by applying the proposed fuzzy controller are very good, in comparison with the fuzzy controller in [26]. However the IAU criterion is relatively increasing, because the control effort in the proposed scheme is important in the beginning period.

\subsection{Example 2}

In this section, we present simulation results showing the tracking performances of the proposed fuzzy adaptive controller applied to an inverted pendulum system.

Let $x_{1}=\theta$ be the angle of the pendulum with respect to the vertical line and $x_{2}=\dot{\theta}$. The dynamic equations of such a system are given by [3]

$\left[\begin{array}{l}\dot{x}_{1} \\ \dot{x}_{2}\end{array}\right]=\left[\begin{array}{ll}0 & 1 \\ 0 & 0\end{array}\right]\left[\begin{array}{l}x_{1} \\ x_{2}\end{array}\right]+\left[\begin{array}{l}0 \\ 1\end{array}\right]\left(f\left(x_{1}, x_{2}\right)+g\left(x_{1}, x_{2}\right) u+d(t)\right)$,

$y=\left[\begin{array}{ll}1 & 0\end{array}\right]\left[\begin{array}{l}x_{1} \\ x_{2}\end{array}\right]$

with

$f\left(x_{1}, x_{2}\right)=\frac{m l x_{2} \sin x_{1} \cos x_{1}-(M+m) G \sin x_{1}}{m l \cos ^{2} x_{1}-(4 / 3) l(M+m)}$,

$g\left(x_{1}, x_{2}\right)=\frac{-\cos x_{1}}{m l \cos ^{2} x_{1}-(4 / 3) l(M+m)}$,

where $G$ is the acceleration due gravity, $M$ is the mass of the cart, $m$ is the mass of the pole, $l$ is the half-length of pole and $u$ is the applied force. It is assumed that the external disturbance $d(t)$ is a square wave having an amplitude \pm 1 with a period of $2 \pi(s)$. The system parameters are given as $M=1 \mathrm{~kg}, \quad m=0.1 \mathrm{~kg}$, $l=0.5 \mathrm{~m}, \quad G=9.8 \mathrm{~m} / \mathrm{s}^{2}$.

The control objective is to force the system output $y$ to track the reference signal $y_{r}=\sin (t)$. We assume that the functions $f(x)$ and $g(x)$ are completely unknown by the controller and only the system output $y$ is available for measurement. In fact, the model (59) is only required for simulation purposes. 
a

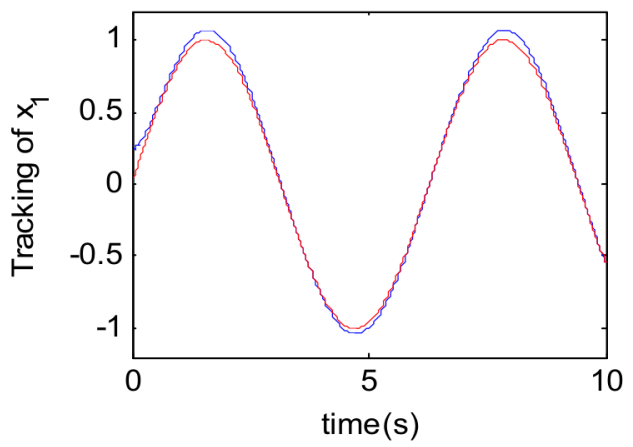

$\mathrm{C}$

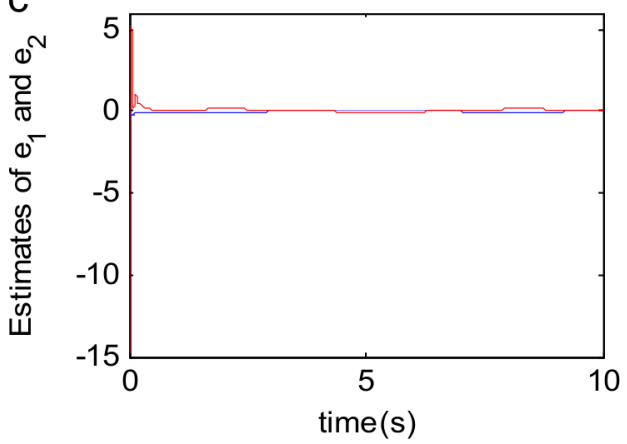

b

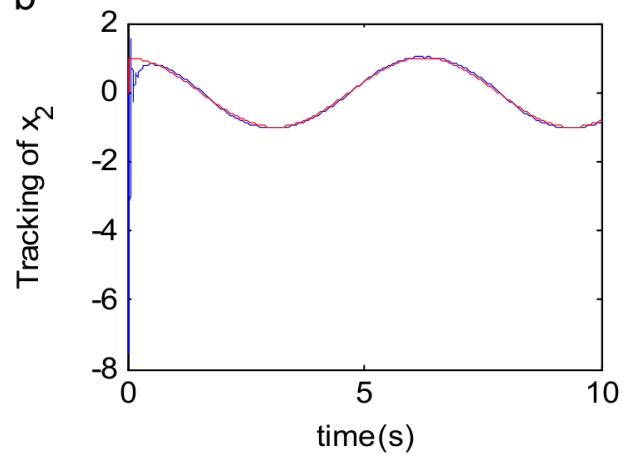

d

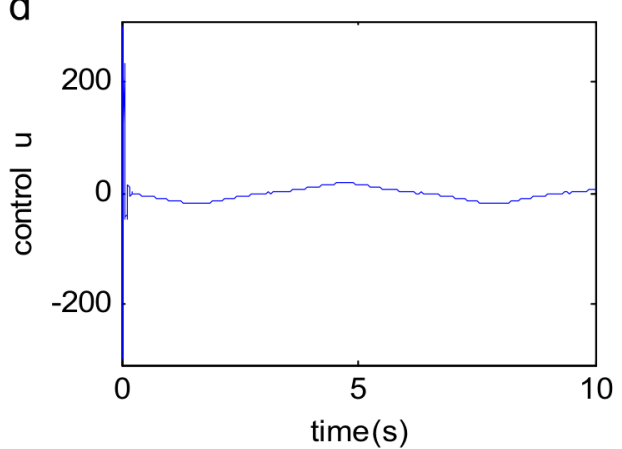

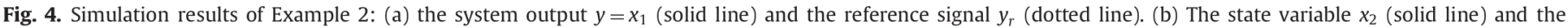
reference signal $\dot{y}_{r}$ (dotted line). (c) The tracking-errors estimates $\hat{e}_{1}$ (dotted line) and $\hat{e}_{2}$ (solid line). (d) The control signal $u$.

Table 3

IAE, ISE and IAU criteria for this proposed method and that of [26] (for Example 2).

\begin{tabular}{llll}
\hline Control method & IAE & ISE & IAU \\
\hline The proposed fuzzy control method & 0.3647 & 0.02654 & 109.1 \\
The fuzzy control method of [26] & 0.4798 & 0.04118 & 105.1 \\
\hline
\end{tabular}

The observer-gain vector $K_{o}$, the feedback-gain vector $K_{c}$, the SPR filter $T(s)$ and the matrix $Q$ are all selected as in the previous example.

The design parameters are selected as $\gamma_{f}=10^{3}, \gamma_{g}=0.2, \gamma_{k}=$ $500, \gamma_{\delta}=10^{-5}, \gamma_{r}=2, \sigma_{f}=8 \times 10^{-3}, \sigma_{g}=10^{-2}, \sigma_{k}=10^{-3}, \sigma_{\delta}=$ $10^{-7}, \delta_{1}=10^{-2}, \delta_{2}=1$ and $\delta_{3}=5$.

The fuzzy membership functions are defined for the variables $\hat{x}_{1}, \hat{x}_{2}$ as follows:

$\mu_{A_{j}^{1}}\left(\hat{x}_{j}\right)=\frac{1}{1+\exp \left(3\left(\hat{x}_{j}+0.25\right)\right)}, \quad \mu_{A_{j}^{2}}\left(\hat{x}_{j}\right)=\exp \left(-\frac{1}{2}\left(\frac{\hat{x}_{j}^{2}}{0.6}\right)\right), \quad$ and

$\mu_{A_{j}^{3}}\left(\hat{x}_{j}\right)=\frac{1}{1+\exp \left(-3\left(\hat{x}_{j}-0.25\right)\right)}$,

The initial conditions are chosen as $x(0)=\left[x_{1}(0), x_{2}(0)\right]^{T}=[0.5,0]^{T}$, $\hat{e}(0)=\left[\hat{e}_{1}(0), \hat{e}_{2}(0)\right]^{T}=[0,1]^{T}, \delta_{4}(0)=1.2$ and $\kappa(0)=[0,0,0,0,0]^{T}$. The elements of $\theta_{f}(0)$ are randomly selected in the interval $[-1,1]$. As well, the elements of $\theta_{g}(0)$ are randomly selected in the interval $[0.4,1.2]$.

The simulation results are depicted in Fig. 4. From this figure, we can see that the system tracks its desired trajectories and the control signal is bounded.

The fuzzy adaptive control method proposed in [26] is also simulated and compared to represent the efficiency of our method proposed in this paper. The results of these qualitative comparison are illustrated in Table 3. It is clear from this table that the tracking performances are better than those in the previous work [26].

\section{Conclusion}

In this paper, an observer-based fuzzy indirect adaptive controller for a class of SISO nonlinear systems has been presented. In the controller designing, neither measurement of the system states nor knowledge of the system nonlinearities is required. Indeed, an observer has been constructed to estimate the tracking error vector and an adaptive fuzzy system has been used to approximate the system nonlinearities. Using the SPR condition and Lyapunov theory, the stability of the closed-loop system has been rigorously proven. Simulation results have been reported to emphasize the performances of the proposed controller.

\section{References}

[1] K. Hornik, Multilayer feedforward networks are universal approximators Neural Netw. 2 (1989) 359-366.

[2] L.X. Wang, J.M. Mendel, Fuzzy basis functions, universal approximation, and orthogonal least square learning, IEEE Trans. Neural Netw. 3 (5) (1992) 807-814.

[3] L.X. Wang, Adaptive Fuzzy Systems and Control: Design and Stability Analysis, Prentice-Hall, Englewood Cliffs, NJ, 1994.

[4] Y.C. Chang, Adaptive fuzzy-based tracking control for nonlinear SISO systems via VSS and $H^{\infty}$ approaches, IEEE Trans. Fuzzy Syst. 9 (2001) 278-292.

[5] B.S. Chen, C.H. Lee, Y.C. Chang, $\mathrm{H}^{\infty}$ Tracking design of uncertain nonlinear SISO systems: adaptive fuzzy approach, IEEE Trans. Fuzzy Syst. 4 (1) (1996) 32-43.

[6] J.T. Spooner, K.V. Passino, Stable adaptive control using fuzzy systems and neural networks, IEEE Trans. Fuzzy Syst. 4 (3) (1996) 339-359.

[7] C.Y. Sue, Y. Stepanenko, Adaptive control of a class of nonlinear systems with fuzzy logic, IEEE Trans. Fuzzy Syst. 2 (4) (1994) 285-294.

[8] S. Tong, X. He, H. Zhang, A combined backstepping and small-gain approach to robust adaptive fuzzy output feedback control, IEEE Trans. Fuzzy Syst. 17 (5) (2009) 1059-1069.

[9] S. Tong, Y. Li, Observer-based fuzzy adaptive control for strict-feedback nonlinear systems, Fuzzy Sets Syst. 160 (12) (2009) 1749-1764.

[10] S. Tong, C. Liu, Y. Li, Fuzzy adaptive decentralized output-feedback control for large-scale nonlinear systems with dynamical uncertainties, IEEE Trans. Fuzzy Syst. 18 (5) (2010) 845-861.

[11] M. Wang, X.P. Liu, P. Shi, Adaptive neural control of pure-feedback nonlinear time-delay systems via dynamic surface technique, IEEE Trans. Syst. Man Cybern. part B: Cybern. 41 (6) (2011) 1681-1692. 
[12] W. Chen, L. Jiao, R. Li, J. Li, Adaptive backstepping fuzzy control for nonlinearly parameterized systems with periodic disturbances, IEEE Trans. Fuzzy Syst. 18 (4) (2010) 674-685.

[13] W. Chen, L. Jiao, J. Li, R. Li, Adaptive NN backstepping output-feedback control for stochastic nonlinear strict-feedback systems with time-varying delays, IEEE Trans. Syst. Man Cybern. Part B: Cybern. 40 (3) (2010) 939-950.

[14] T. Zhang, S.S. Ge, C.C. Huang, Adaptive neural network control for strictfeedback nonlinear systems using backstepping design, Automatica 36 (12) (2000) 1835-1846.

[15] S.S. Ge, C. Wang, Direct adaptive NN control for a class of nonlinear systems, IEEE Trans. Neural Netw. 13 (1) (2002) 214-221.

[16] W.S. Chen, Adaptive backstepping dynamic surface control for systems with periodic disturbances using neural networks, IET Control Theory Appl. 3 (10) (2009) 1383-1394.

[17] D. Wang, Neural network-based adaptive dynamic surface control of uncertain nonlinear pure-feedback systems, Int. J. Robust Nonlinear Control 21 (2011) 527-541.

[18] Y.G. Leu, T.T. Lee, W.Y. Wang, Observer-based adaptive fuzzy-neural control for unknown nonlinear dynamical systems, IEEE Trans. Syst. Man Cybern. Part B: Cybern. 29 (1999) 583-591.

[19] Y.G. Leu, W.Y. Wang, T.T. Lee, Observer-based direct adaptive fuzzy-neural control for nonaffine nonlinear systems, IEEE Trans. Neural Netw. 16 (4) (2005) 853-861.

[20] H.X. Li, S. Tong, A hybrid fuzzy adaptive control for a class of nonlinear MIMO systems, IEEE Trans. Fuzzy Syst. 11 (1) (2003) 24-34.

[21] S. Tong, H.X. Li, W. Wang, Observer-based adaptive fuzzy control for SISO nonlinear systems, Fuzzy Sets Syst. 148 (2004) 355-376.

[22] W.Y. Wang, Y.G. Leu, T.T. Lee, Output-feedback control of nonlinear systems using direct adaptive fuzzy-neural controller, Fuzzy Sets Syst. 140 (2003) 341-358.

[23] Y.J. Liu, S.C. Tong, W. Wang, Y.M. Li, Observer-based direct adaptive fuzzy control of uncertain nonlinear systems and its applications, Int. J. Control Autom. Syst. 7 (4) (2009) 681-690.

[24] H.F. Ho, Y.K. Wong, A.B. Rad, W.L. Lo, State observer based indirect adaptive fuzzy tracking control, Simul. Modell. Pract. Theory 13 (2005) 646-663.

[25] J.H. Park, G.T. Park, S.H. Kim, C.J. Moon, Output feedback control of uncertain nonlinear systems using a self-structuring adaptive fuzzy observer, Fuzzy Sets Syst. 151 (2005) 21-42.

[26] A. Boulkroune, M. Tadjine, M. M'Saad, M. Farza, How to design a fuzzy adaptive control based on observers for uncertain affine nonlinear systems, Fuzzy Sets Syst. 159 (2008) 926-948.

[27] A. Boulkroune, M. Tadjine, M. M'Saad, M. Farza, Adaptive fuzzy controller for non-affine systems with zero dynamics, Int. J. Syst. Sci. 40 (4) (2009) 367-382.

[28] C.H. Wang, T.C. Lin, T.T. Lee, H.L. Liu, Adaptive hybrid intelligent control for uncertain nonlinear dynamical systems, IEEE Trans. Syst. Man Cybern. Part B: Cybern. 32 (5) (2002) 583-597.

[29] A. Boulkroune, M. Tadjine, M. M'Saad, M. Farza, Adaptive fuzzy observer for uncertain nonlinear systems, Control Intell. Syst. 39 (3) (2011) 145-150.

[30] C.M. Lin, C.F. Hsu, Adaptive fuzzy sliding-mode control for induction servomotor systems, IEEE Trans. Energy Convers. 19 (2) (2004) 362-368.

[31] M. Roopaei, M.Z. Jahromi, Synchronization of two different chaotic systems using novel adaptive fuzzy sliding mode control, Chaos 18 (2008) 033133.

[32] A. Boulkroune, M. M'Saad, A practical projective synchronization approach for uncertain chaotic systems with dead-zone input, Commun. Nonlinear Sci. Numer. Simulat. 16 (2011) 4487-4500.

[33] Y.H. Kim, F.L. Lewis, C.T. Abdallah, A dynamic recurrent neural-network-based adaptive observer for a class of nonlinear systems, Automatica 33 (8) (1997) 1539-1543.

[34] A. Poursamad, A.H. Davaie-Markazi, Robust adaptive fuzzy control of unknown chaotic systems, Appl. Soft Comput. 9 (2009) 970-976.

[35] M.K. Ciliz, Combined direct and indirect adaptive control for a class of nonlinear systems, IET Control Theory Appl. 3 (1) (2009) 151-159.

[36] A. Isidori, Nonlinear Control Systems, Springer-Verlag, Berlin, Germany, 1995.

[37] T.P. Zhang, S.S. Ge, Adaptive neural control of MIMO nonlinear state timevarying delay systems with unknown dead-zones and gain signs, Automatica 43 (2007) 1021-1033.

[38] S.S. Ge, C. Wang, Adaptive neural control of uncertain MIMO nonlinear systems, IEEE Trans. Neural Netw. 15 (2004) 674-692.

[39] H. Zhang, Z. Bien, Adaptive fuzzy control of MIMO nonlinear systems, Fuzzy Sets Syst. 115 (2000) 191-204.

[40] A. Boulkroune, M. M'Saad, M. Tadjine, M. Farza, Fuzzy adaptive controller for MIMO nonlinear systems with known and unknown control direction, Fuzzy Sets Syst. 161 (2010) 797-820.

[41] A. Boulkroune, M. M'Saad, On the design of observer based fuzzy adaptive controller for nonlinear systems with unknown control gain sign, Fuzzy Sets Syst. 201 (2012) 71-85.

[42] A. Boulkroune, M. M'Saad, A fuzzy adaptive variable-structure control scheme for uncertain chaotic MIMO systems with sector nonlinearities and deadzones, Expert Syst. Appl. 38 (12) (2011) 14744-14750.

[43] A. Boulkroune, M. M'Saad, Fuzzy adaptive observer-based projective synchronization for nonlinear systems with input nonlinearity, J. Vib. Control 18 (3) (2012) 437-450.

[44] J.E. Slotine, W. Li, Applied Nonlinear Control, Prentice-Hall, Englewood Cliffs, NJ, 1991.

[45] M. French, E. Rogers, Approximate models for adaptive feedback linearization, Int. J. Control 68 (6) (1997) 13057-13121.
[46] N. Kim, A.J. Calise, Several extensions in methods for adaptive output feedback control, IEEE Trans. Neural Netw. 18 (2) (2007) 482-494.

[47] S. Sastry, A. Isidori, Adaptive control of linearizable systems, IEEE Trans. Autom. Control 34 (11) (1989) 1123-1131.

[48] S. Sastry, M. Bodson, Adaptive Control: Stability, Convergence and Robustness, Prentice Hall, Englewood Cliffs, NJ, 1989.

[49] D.Y. Meddah, A. Benallegue, A.R. Cherif, A neural network robust controller for a class of nonlinear MIMO systems, in: Proceedings of the 1997 IEEE Intemational Conference on Robotics and Automation, Albuquerque, New Mexico, April 1997, pp. 2645-2650.

[50] H.A. Youcef, M.A. Wahba, Adaptive fuzzy mimo control of induction motors, Expert Syst. Appl. 36 (2009) 4171-4175.

[51] C.H. Chen, C.M. Lin, T.Y. Chen, Intelligent adaptive control for MIMO uncertain nonlinear systems, Expert Syst. Appl. 35 (2008) 865-877.

[52] G. Ablay, Sliding mode control of uncertain unified chaotic systems, Nonlinear Anal.: Hybrid Syst. 3 (4) (2009) 531-535.

[53] G. Chen, X. Dong, On feedback control of chaotic continuous-time systems, IEEE Trans. Circuits Syst. I 40 (1993) 591-600. 